\title{
Postexposure prophylaxis with oseltamivir reduced influenza transmission in households
}

Hayden FG, Belshe R, Villanueva C, et al. Management of influenza in households: a prospective, randomized comparison of oseltamivir treatment with or without postexposure prophylaxis. J Infect Dis 2004;189:440-9.

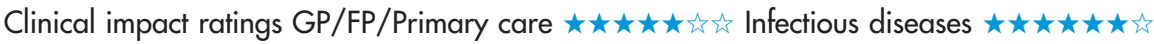

In household contacts (HHCs) (after index influenza [flu] patients received oseltamivir), is postexposure prophylaxis (PEP) more effective than oseltamivir treatment at the time of illness (expectant treatment) for preventing flu transmission?

METHODS

L

Design: cluster (household level) randomised controlled trial

Allocation: $\{$ concealed $\} \dagger$.

Blinding: unblinded.*

Follow up period: 30 days.

Setting: households in Europe and North America.

응 Participants: $812 \mathrm{HHCs}$ (age range 1-83 y, 55\% girls/women) of index patients with a flu like illness during a community flu outbreak. Eligible households had 3-8 members, including $\geqslant 1$ index patient and $\geqslant 2$ eligible contacts $\geqslant 1$ year of age. Exclusion criteria included pregnancy, breastfeeding, and cancer. households with $410 \mathrm{HHCs}$ ) or expectant treatment $(n=139$ households with $402 \mathrm{HHCs}$ ). All index patients and $\mathrm{HHCs}$ developing illness in the expectant treatment group received oseltamivir treatment (adults and adolescents $75 \mathrm{mg}$ capsules; and children 30-60 mg suspension twice/day) for 5 days. HHC in the PEP group received the same dosage as for treatment but given once/day.

Outcome: number of households with $\geqslant 1$ secondary patient who had laboratory confirmed flu during the 10 day period after the start of treatment in the index patient.

*See glossary.

tInformation provided by author.
D Intervention: households were allocated to PEP $(n=138$

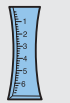

Patient follow-up: $97 \%$ (intention to treat analysis).

\section{MAIN RESULTS}

Fewer households in the PEP group than in the expectant treatment group had $\geqslant 1$ secondary contact with laboratory confirmed flu (table).

\section{CONCLUSION}

In household contacts (after treatment of all index influenza [flu] patients with oseltamivir), postexposure prophylaxis was more effective than treatment with oseltamivir at the time of developing illness for reducing flu transmission in households.

\section{Abstract and commentary also appear in ACP Journal Club.}

\section{Commentary}

Tred he study by Hayden et al showed that oseltamivir is effective for reducing flu transmission from an infected family member to others in the household. The efficacy was greatest in a subgroup of HHCs who were not infected at baseline (relative risk reduction or protective efficacy $79 \%, 95 \% \mathrm{Cl} 41$ to 92 , number needed to treat 6 ) compared with that in the overall intention to treat population (table)

A number of other practical considerations are worth noting about the clinical use of oseltamivir or zanamivir (another neuraminidase inhibitor with proven effectiveness). Firstly, the avian flu, which has peppered most countries in southeast Asia and China, should be susceptible to these 2 drugs, and their use could have reduced the corresponding mortality and morbidity that occurred. Secondly, the severe acute respiratory syndrome, caused by a variant of the coronavirus group, may be difficult to distinguish from flu. Both can occur at the same time in a population. It is important to note that flu is treatable whereas the severe acute respiratory syndrome is not.

Neuraminidase inhibitors should be part of a comprehensive flu prevention and treatment program. The drugs are not just for the elderly, but for the whole population. We are all "at risk" of sinusitis, a prolonged illness with loss of time from work and possibly death. However, it is important to remember that as good as these drugs are, they are a supplement to flu vaccination. The flu vaccine has been shown to have a $50-90 \%$ protective efficacy depending on the vaccine strain and population group. ' The vaccine also reduces hospitalization for pneumonia as well as reducing the risk of heart failure, stroke, and death from all causes-not insignificant positive side effects.

Peter A Gross, MD Hackensack University Medical Centre and University of Medicine and Dentistry of New Jersey-New Jersey Medical School Hackensack, New Jersey, USA For correspondence: Professor F G Hayden, University of Virginia School of Medicine, Charlottesville, VA, USA. fgh@virginia.edu

Source of funding: Hoffman-La Roche, Inc.

Postexposure prophylaxis $v$ expectant treatment with oseltamivir at the time of developing influenza in household contacts (after treatment of all index influenza patients with oseltamivir)*

\begin{tabular}{llllll}
\hline Outcomes at 10 days & Household population & $\begin{array}{l}\text { Post exposure } \\
\text { prophylaxis }\end{array}$ & $\begin{array}{l}\text { Expectant } \\
\text { treatment }\end{array}$ & RRR (95\% Cl) \\
\hline Households with $\geqslant 1$ secondary & All & $7.4 \%$ & $19.9 \%$ & $63 \%(27$ to 81$)$ \\
patient with confirmed influenza & Index patient had LCl & $10.7 \%$ & $25.8 \%$ & $59 \%$ (18 to 80) \\
\hline
\end{tabular}

* $\mathrm{LCl}=$ laboratory confirmed influenza. Other abbreviations defined in glossary; RRR, NNT, and Cl calculated from data in article

†NNT refers to number of households needed to treat. 